\title{
Seasonal polar cap radiation zones in dayside magnetosphere
}

\author{
G. Pugacheva ${ }^{1}$, A. A. Gusev ${ }^{2,3}$, U. B. Jayanthi ${ }^{2}$, N. J. Schuch ${ }^{1}$, and W. N. Spjeldvik ${ }^{4}$ \\ ${ }^{1}$ Southern Regional Space Research Center/INPE, Santa Maria, RS, Brazil \\ ${ }^{2}$ National Institute for Space Research, INPE, Sao Jose dos Campos, SP, Brazil \\ ${ }^{3}$ Space Research Institute of Russian Academy of Science, Moscow, Russia \\ ${ }^{4}$ Weber State University, Utah, Ogden, USA
}

(Received April 8, 2004; Revised June 4, 2004; Accepted June 8, 2004)

\begin{abstract}
The phenomenon of quasi-stable trapping of charged particles in the $\mathrm{keV}$ to $\mathrm{MeV}$ energy range within the polar cusp region of the Earth's magnetosphere is explored. The remote equatorial magnetic field lines on the dayside magnetosphere are compressed by the solar wind and exhibit two local minima in the geomagnetic field strength along the field line in high latitudes. These minima, on both sides of the equator, result in stable confinement structures. Numerical modeling of charged particle orbits that pass through the regions of these local field minima has been carried out using different seasonal Earth tilt and different magnetospheric disturbance level. These orbit tracings show when and where these off-equatorial trapped radiation zones would be situated. The existence and extent of these confinement zones depend on the tilt angle. Indeed, the northern cusp confinement zone appears only at the northern summer solstice, while the southern cusp particle capture zone appears around winter solstice. The particle orbits that pass through opposite off-equatorial field minimum during solstices reveal a bound of the geomagnetic equatorial plane on the day sector. During equinox, the particle confinement zones exist in both cusps at times of disturbed magnetosphere conditions. The trapped particles drift within the trapping zones with periods of the several minutes, conserving the 1 st and 2 nd adiabatic invariants.
\end{abstract}

Key words: Magnetosphere, trapped particles, radiation belt, cusp.

\section{Introduction}

Measurements of energetic protons, ions and relativistic electrons with POLAR (Chen et al., 1997; Sheldon et al., 1998) and INTERBALL (Pissarenko et al., 2001) satellites have shown that there exist zones with trapped radiation in the dayside magnetosphere at high latitudes. A possible mechanism for the formation of the near cusp-region confinement zones was considered more than 30 years ago by Antonova and Shabansky (1968) and Shabansky (1972), and these ideas have been further developed by Antonova et al. (2001, 2003). It is known that the dayside magnetic field under compression by solar wind modifies the near dipolar topology becoming more intense at the geomagnetic equator than in the higher latitude regions, and that occurs on either side of the equator rather near the polar cusps. The behavior of the charged particles in the dayside magnetosphere has been analyzed by Delcourt and Sauvaud $(1998,1999)$ by exploring the single charged particle trajectories utilizing the Tsyganenko geomagnetic field model (Tsyganenko, 1989) with geoelectric field effects. These authors showed that in the presence of two localized magnetic minima, a charged particle initially mirroring within the geomagnetic equator plane can deviate abruptly under the cuspward mirror force to one of the minima when it enters the dayside sector during longitudinal drift motion. This motion may give rise to the presence of hundreds of $\mathrm{keV}$ particle population in the higher

Copy right (c) The Society of Geomagnetism and Earth, Planetary and Space Science (SGEPSS); The Seismological Society of Japan; The Volcanological Society of Japan; The Geodetic Society of Japan; The Japanese Society for Planetary Sciences; TERRAPUB latitudes, something that is observed experimentally. In the present work we study a possibility of the formation of the confinement zones in the cusp region based on the topology of the empirical Tsyganenko geomagnetic field model and the Volland-Stern electric field model (Volland, 1978). We here for the first time show that such particle confinement zones can exist in determined conditions which depend on the tilt value and on the geomagnetic activity level. It results in noticeable seasonal variations of the near-cusp radiation belt parameters.

\section{The Dayside Magnetic Field Line Topology}

We analyzed the dayside geomagnetic field line topology at three special time periods characterized by inclination of the Earth's rotation axis to the Sun direction: summer and winter solstices (for northern hemisphere) and spring equinox. The Earth's tilt is in the range of $55.5^{\circ}-77^{\circ}$ around summer solstice, of $79^{\circ}-101^{\circ}$ at equinox time, and of $102^{\circ}-$ $124^{\circ}$ in winter solstice. The building of the geomagnetic field lines was done utilizing the code provided in the Tsyganenko (1989) model package. Figure 1 depicts the magnetic field strength versus geodetic latitudes along the dayside field lines of the Greenwich meridian for spring equinox for quiet magnetosphere conditions. This figure shows the existence of two off-equatorial minima in the magnetic field strength. The field lines are anchored to various latitudes of the Earth's surface. Those that are anchored to the lower latitudes $\left(\right.$ Lat. $=70^{\circ}$ ) exhibit only one traditional field minimum at the regular equatorial plane. Lines with two off-equatorial minima are anchored to $73^{\circ}-80^{\circ}$ latitudes in the quiet mag- 


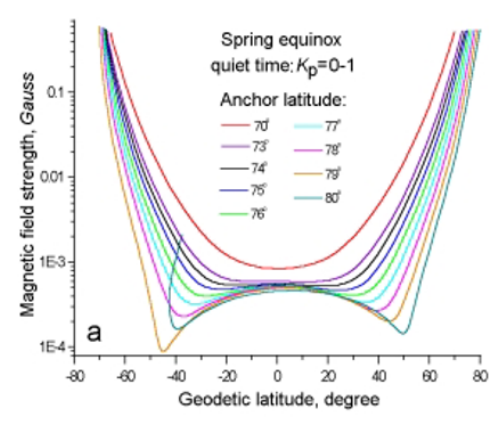

Fig. 1. The geomagnetic field strength along dayside field lines versus geographic latitudes for the different anchor latitudes in spring equinox in the quiet magnetosphere.

netosphere at equinox time. The two-minima structure shifts inwards into the magnetosphere, placing between $71^{\circ}$ and $75^{\circ}$ latitudes when $K_{p}=8$. Similar topology of the magnetic field lines with two off-equatorial minima are found during summer and winter solstices both in the quiet and disturbed magnetosphere. Magnetic topology with two distinct minima in field strength is also found at all meridians from $0^{\circ}$ to $360^{\circ}$ when they are exposed to the dayside. Thus the two minima structures exist for any relative Earth axial tilt both during quiet and disturbed conditions. Each one of the two distinct magnetic field minima can cause local magnetic trapping in the both hemispheres, exactly the same way as the one geomagnetic field main minimum belonging to deeper geomagnetic lines, and located in the geomagnetic equatorial plane causes the powerful and dangerous phenomenon of the Earth's radiation belts.

\section{Particle Motion in the Geomagnetic and Geo- electric Fields}

To answer on the question of localized trapped radiation, we need to review the main mechanism of the radiation belt formation. Charged particles drift around the Earth under the force of geomagnetic field gradient, primarily on concentric circular trajectories around the dipole center pass through the magnetic field minimum in the geomagnetic equatorial plane. The particles execute thousands of rotations around the Earth, thus becoming stably trapped in that field mini- mum, and their flux is accumulated in this geomagnetic trap with the coefficient of accumulation reaching several millions. This mechanism provides, for example, the great 10 $\mathrm{MeV}$ trapped proton flux of about $10^{5}-10^{6} \mathrm{~cm}^{-2} \mathrm{~s}^{-1}$ in the belt maximum at $L \sim 1.5$, a flux which could impact a lethal doze on a human being in Earth orbit at this location over several minutes.

Finding the dayside off-equatorial field minima (Fig. 1) we intend to search, if analogously to radiation belts, these minima might indeed correspond to some closed cusp confinement zones. If a charged particle passing along the field minima illustrated in Fig. 1, returns to the same point where it starts, it means that the particle could be trapped in the region. We analyzed a topology of the capture region with the method of single charged particle orbit tracing in a specified magnetic field topology. The orbit simulation was based on the numerical solution of the Lorentz equation (Eq. (1)) for a particle motion in the geomagnetic $B$ and the geoelectric $E$ fields:

$$
\frac{d(m \vec{V})}{d t}=q\left(\vec{E}+\frac{1}{c} \vec{V} \times \vec{B}\right)
$$

where $q, m$, and $V$ are particle charge, relativistic mass, and velocity, and $c$ is the light velocity. For the geomagnetic field structure we use the Tsyganenko geomagnetic field model. The electric fields considered both the corotation and the convection fields in the equatorial plane with corotation field potential $U_{\text {cor }}=-C R_{e} / R$, here $C=91.5 \mathrm{kV}$ (Roederer, 1970), and the convection field potential $U_{\mathrm{V}-\mathrm{S}}=-A R^{2} \sin \phi$, of Volland-Stern model with coefficient $A$ dependent on geomagnetic activity $A=$ $0.0449 /\left(1 .-0.159 K_{P}+0.009 K_{P}^{2}\right)^{3}$, in units of $\mathrm{kV} / R_{e}^{2}$, and where $\phi$ is the azimuthal angle between the direction of the field vector and the sunward axis. $R$ is the radial distance from magnetic dipole center.

The electric field structure away from the Earth's equatorial plane is much less well known. In the 3-D modeling, we assume the geomagnetic field lines are equipotential with the electric potential equal to that at the point of field line crossing geomagnetic equatorial plane. Tracing the potential along the neighboring magnetic field lines, one can compute the electric field vector as $E=-\operatorname{grad} U$. We do not detail these calculations here because we further specialize
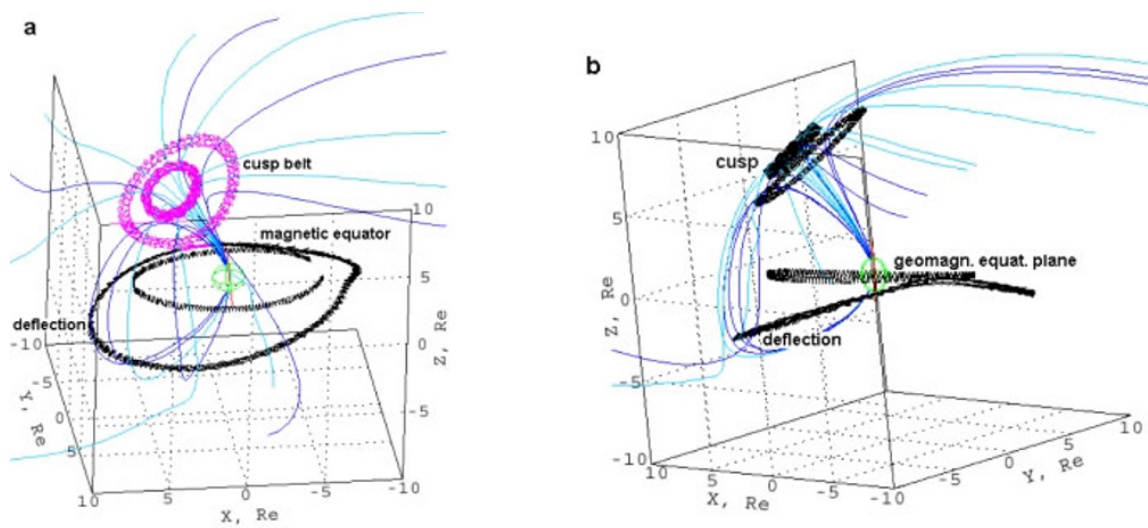

Fig. 2. The 3-Dim images $(\mathrm{a}, \mathrm{b})$ in GSM coordinates of the $0.3 \mathrm{MeV}$ proton orbit starting from the northern field minimum (anchor latitude $\left.75^{\circ}\right)$ in summer solstice in the disturbed magnetosphere. The orbit "deflection" belongs to proton passing the southern field minimum at relatively low $L=8-9$. 


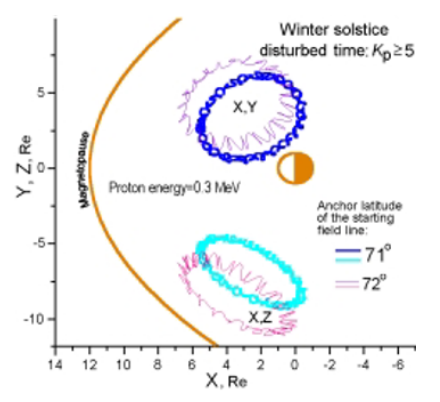

Fig. 3. The $\mathrm{X}, \mathrm{Y}$ and $\mathrm{X}, \mathrm{Z}$ projections of the $0.3 \mathrm{MeV}$ proton orbits passing the southern field minimum in winter solstice in disturbed magnetosphere.

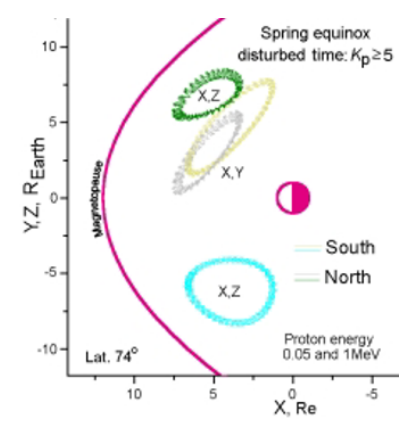

Fig. 4. The $\mathrm{X}, \mathrm{Y}$ and $\mathrm{X}, \mathrm{Z}$ projections of the proton orbits passing the northern and southern field line minima in spring equinox in disturbed magnetosphere.

to analyze the orbits of protons with energies great enough ( 0.1 to several $\mathrm{MeV}$ ) such that they drift primarily because of gradient- $B$ effect, rather than $E \times B$ effects.

Equation (1) is solved numerically applying the RungeKutta-Guills method. The corresponding Fortran code uses double precision, and where necessary even quadruple precision (e.g., Gusev and Pugacheva, 1982). In this case, unlike cosmic ray calculations, it was not necessary to recheck the solution by computing reversed trajectories because the trapped particles return to the initial point in performing finite motion.

\section{Confinement Zones of Trapped Radiation in the Cusp Region}

Our task was to study the characteristic features of possible particle confinement zones in the cusp regions, utilizing the reliable empirical geomagnetic tilted field model. The interest here is to know the morphological and confinement details of the population in these regions at quite and disturbed conditions. We first consider the influence of geomagnetic axis inclination to the Sun-Earth direction on the particle capture in the cusp. We simulated trajectories of protons with energies from $100 \mathrm{keV}$ to $2 \mathrm{MeV}$ starting from the northern $B$-field minimum of each field line, similar to Fig. 1, for summer solstice with initial condition $(V \cdot B)=0$. Figure 2 shows projections of the various 3-Dim drift trajectories of protons confined in the summer solstice $B$-field topology in the northern minimum region for the geomagnetic disturbed times. It can be seen that the orbits are finite, circular concentric trajectories along the minima associated with the field lines anchored to $74^{\circ}$ and $78^{\circ}$ latitudes of the

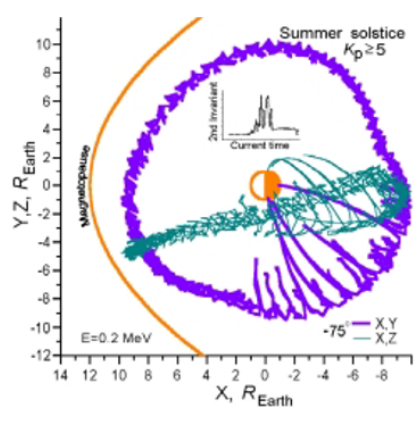

Fig. 5. The $\mathrm{X}, \mathrm{Y}$ and $\mathrm{X}, \mathrm{Z}$ projections of the proton orbit starting in summer solstice from the southern field minimum at $L \cong 10-12$ showing a violation of 2 nd adiabatic invariant.

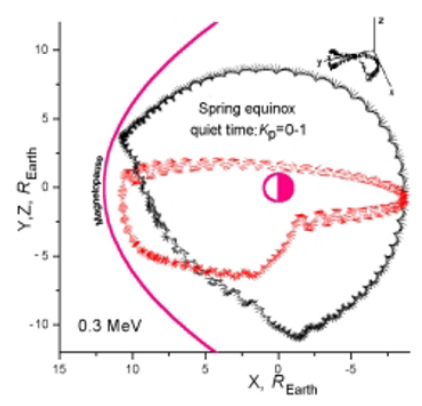

Fig. 6. The X, Y; X, Z projections and 3D image (right corner) of the proton orbit starting from the northern and southern (right corner) off-equatorial field minimum in the equinox in the quiet magnetosphere.

Greenwich meridian. The protons keep pitch-angle near $90^{\circ}$ and thus with the 2 nd adiabatic invariant, $J=0$, conserved. These protons occupy the sub polar, cusp region in a form of a plain belt. The $1 \mathrm{MeV}$ proton drift period around cusp is about several minutes. Indeed, it is a confinement zone of energetic charged particles in the cusp region which could be named a cusp radiation belt of the Earth.

During quiet times, the particle belt belongs to the more distant field lines. During disturbed conditions, its width spreads out to about 5 latitudinal degrees. In contrast, the protons passing through the southern hemisphere minimum do not create a confinement zone around the southern cusp (see Fig. 2, curve "deflection"), but drift around the entire Earth as the usual main radiation belt particles. The drift period around the cusp region is several minutes and they can drift for many orbits within the belt, thus accumulating flux until some dynamical process (there are many in the polar region) precipitates them out from these fairly stable orbits. In the northern summer solstice period, this polar belt appears only in the northern hemisphere. Our simulations show that the $2 \mathrm{MeV}$ proton orbit is still closed, while $3 \mathrm{MeV}$ protons have sufficiently large gyroradii to precipitate out of the region. One thus recognizes that even the greatest electric field values provided from the Volland-Stern model with $K_{p}=8$ do not significantly affect the orbits of energetic $0.1-2 \mathrm{MeV}$ protons (at $L=8-10$ ) in the equatorial plane. Figure 3 demonstrates that the rather similar structure of energetic particle confinement zone occurs only at the southern cusp (see X, Z projection) around winter solstice during magnetosphere active periods.

During spring equinox in a disturbed magnetosphere there 
appear two cusp confinement zones (Fig. 4) where energetic protons are confined in both the northern and the southern off-equatorial field minima. During quiet time the cusp belts practically do not exist at equinox. The influence of the geoelectric field on the particle capture in the cusps was evaluated with simultaneous greatest electric field allowed by the Volland-Stern model, with $K_{p}=8$, and also with the geomagnetic field with IOPT $=8$ (according to the Tsyganenko model). The stable charged particle confinement at $\mathrm{keV}$ and $\mathrm{MeV}$ energies was still observed. An electric field influence needs further study, especially for low energy particles.

\section{Deflection of the Geomagnetic Equatorial Plane under the Solar Wind Pressure}

The simulated behavior of energetic protons during summer solstice has showed the possibility of charged particle confinement around the northern off-equatorial field minimum. We further studied what happens with the hundreds $\mathrm{keV}$ protons starting their drift orbits from another offequatorial field minimum in the southern hemisphere, carried out with the same condition $(V \cdot B)=0$. These energetic proton trajectories are shown in Figs. 5 and 6 . We find that the trajectories do not create confinement zone around southern cusp, and rather they drift around the whole Earth in geomagnetic equatorial plane as usual radiation belt particles. However, the plane is deflected on the dayside (Fig. 2, curve labeled "deflection"), and deflection angle does depend on the $K_{p}$-index. It looks as the geomagnetic equatorial plane is broken and deflected for particles drifting around the Earth along the southern field minimum.

This effect occurs only on the distant peripheral regions of the magnetosphere at $L$-shells of about $8-12$. The protons orbiting in this deflected geomagnetic equator plane at lower $L$-shells of about 8-9 conserve their 2 nd invariant and pitchangle near $90^{\circ}$ (e.g., Fig. 2, curve "deflection"). At higher $L$-shells $(L \sim 10-12)$, strong variations in the 2 nd invariant occur on the night side and after one drift period the particle loses "memory" of its initial 2nd invariant, thus returning to the starting point with the 2 nd invariant changed (i.e., see Fig. 5).

This finding implies that energetic particles can not be trapped on such orbits for more than 2-3 drift rotations, and should well be considered as quasi-trapped. Indeed, in the work of Antonova et al. (2003) it was pointed out that possible violation of the 2 nd adiabatic invariant for particle motion through dayside off-equatorial magnetic field minima can occur.

At quiet times during equinox, the proton orbits passing both the northern and southern $B$-field minima (i.e., Fig. 1) do not show the cusp belt zones, but they drift around the whole Earth and residing in the equatorial plane on the night side and in the perpendicular plane on the dayside (see Fig. 6). The possibility of these kinds of particle trajectories was also described by Delcourt et al. $(1998,1999)$ and was suggested to provide a source of the energetic particle fluxes observed with the POLAR and INTERBALL satellite instruments at high latitudes (i.e., Karra and Fritz, 1999; Kudela et al., 2002).

\section{Conclusion}

Numerical simulation of the energetic proton orbits in the empirical Tsyganenko magnetic field model showed the existence of closed confinement zones in the cusp regions of the distant Earth's magnetosphere. The zones form a kind of a seasonal cusp radiation belt and their location depends on the geomagnetic dipole tilt with the the northern cusp belt existing at summer solstice and the southern cusp belt during winter solstice. At equinox time they exist in both the hemispheres only during disturbed magnetosphere. The cusp belts are very thin in latitude units (several latitudinal degrees). Energetic protons are temporarily trapped there for times at least several minutes up to possibly some days.

At solstices, while in one of these off-equatorial minimum region the cusp contains the trapped radiation, in the other off-equatorial field minimum the particles orbit around the Earth center in a plane deflected on the dayside from the geomagnetic equatorial plane at an angle dependent on the geomagnetic activity index $K_{p}$. At equinox, in the quiet time, the deflection is observed in both hemispheres and the deflection angle is nearly equal to $90^{\circ}$. These modeling results could be useful for the analysis of the observations of the trapped radiation at high latitudes. Further studies of the life time and the accumulation coefficient of the particles in these cusp polar confinement zones and the detail consideration of the magnetospheric electric fields are needed.

\section{References}

Antonova, A. E. and V. P. Shabansky, Structure of geomagnetic field at great distances from the Earth, Geomagn. Aeron., 8, 639, 1968.

Antonova, A. E., Yu. I. Gubar, and A. P. Kropotkin, Energetic trapped particles in a Model Magnetic Field of the magnetospheric cusp, Geomagn. Aeron., 41, n1, 6-9, 2001.

Antonova, A. E., Yu. I. Gubar, and A. P. Kropotkin, Effects in the radiation belts caused by the second adiabatic invariant violation in the presence of dayside off-equatorial magnetic field minima, Adv. Space Res., 31, n5, 1223-1228, 2003.

Chen, J., T. A. Fritz, R. B. Sheldon, et al., A new temporally confined population in the polar cap, Geophys. Res. Lett., 24, 1447, 1997.

Delcourt, D. C. and J.-A. Sauvaud, Recirculation of plasma sheet particles into high latitude boundary layer, J. Geophys. Res., 103(A11), 2652126532, 1998.

Delcourt, D. C. and J.-A. Sauvaud, Populating of cusp and boundary layers by energetic (hundreds of $\mathrm{keV}$ ) equatorial particles, J. Geophys. Res., 104(A10), 22635-22648, 1999.

Gusev, A. A. and G. I. Pugacheva, About output of quasi-trapped electrons from the atmosphere, Geomagn. Aeron., 22, 487-489, 1982.

Karra, M. and T. Fritz, Energy dispersion features in the vicinity of the cusp, Geophys. Res. Lett., 26, 3553, 1999.

Kudela, K., V. N. Lutsenko, et al., High energy particle dispersion events observed by Interball-1,2, Adv. Space Res., 30, 2849, 2002.

Pissarenko, N. F., I. N. Kirpichov, V. N. Lutsenko, et al., Cusp energetic particles observed by Interball Tail Probe in 1996, Phys. Chem. Earth (C), 26, n1-3, 241-245, 2001.

Roederer, J., Dynamics of Geomagnetically Trapped Radiation, SpringerVerlag, Heidelberg-New York, 1970.

Shabansky, V. P., Some processes in magnetosphere, Space Sci. Rev., 12(3), 299-418, 1972.

Sheldon, R. B., H. E. Spence, et al., The discovery of trapped energetic electrons in the outer cusp, Geophys. Res. Lett., 25, 1825, 1998.

Tsyganenko, N. A., Magnetospheric magnetic field with a warped tail current sheet, Space Sci.Rev., 37, 5, 1989.

Volland, H., A model of the magnetospheric electric convection field, $J$. Geophys. Res., 83, 2695, 1978.

G. Pugacheva (e-mail: galina@das.inpe.br), A. A. Gusev, U. B. Jayanthi, N. J. Schuch, W. N. Spjeldvik 